\title{
Functional analysis of the UVR8 photoreceptor from the monocotyledonous Zea mays
}

\author{
María Belén Fernández ${ }^{1} \cdot$ Lorenzo Lamattina $^{1} \cdot$ Raúl Cassia $^{1}$
}

Received: 12 December 2019 / Accepted: 20 June 2020

(c) Springer Nature B.V. 2020

\begin{abstract}
Low UV-B fluence is a signaling stimulus that regulates various physiological processes and induces photomorphogenic responses in plants. The specific UV-B receptor UVR8 is a key component in these processes. Although UVR8 sequence is conserved, few homologs have been cloned and reported to be functional. Here we show the cloning and functional analysis of Zea mays UVR8 (ZmUVR8). ZmUVR8 presents 73\% of identity with AtUVR8, maintaining the key tryptophan responsible of UV-B perception. ZmUVR8 also contains the VP domain, involved in the interaction with the proteins CONSTITUTIVELY PHOTOMORPHOGENIC 1 (COP1) and REPRESSOR OF UV-B PHOTOMORPHOGENESIS 1 (RUP1). Whereas UVR8 was expressed in non-irradiated Arabidopsis and maize leaves, after $2 \mathrm{~h}$ of UV-B irradiation, its expression was reduced. The expression of chalcone synthase $(C H S)$, involved in flavonoid biosynthesis and regulated by UVR8, was increased in irradiated Arabidopsis and maize leaves. Arabidopsis uvr8-1 null mutant was complemented with ZmUVR8 driven by the CaMV-35S promoter and fused to eGFP. ZmUVR8-eGFP fusion was mainly localized in nuclei of transgenic lines, irrespective of UV-B treatments. UV-B suppressed hypocotyl elongation in wild type (WT) Arabidopsis plants, whereas in uvr8-1 hypocotyl growth was observed. However, hypocotyl elongation was reduced in UV-B irradiated transgenic lines complemented with ZmUVR8. Moreover, CHS and transcription factor HY5 (ELONGATED HYPOCOTYL 5) expression were also restored in these plants. These results confirm that ZmUVR8 is similar enough to AtUVR8 to restore UV-B perception and signaling in Arabidopsis mutant $u v r 8-1$, thus being a functional UV-B photoreceptor. That reinforce the importance of UVR8 as a functional UV-B-responsive regulator in land plants.
\end{abstract}

Keywords UVR8 $\cdot$ UV-B $\cdot$ Maize $\cdot$ Monocotyledonous $\cdot$ Arabidopsis

\section{Introduction}

Ultraviolet-B (UV-B) radiation is the region between 280 and $315 \mathrm{~nm}$ of the total Sun's electromagnetic spectrum. Ultraviolet irradiances reaching the surface of the Archean Earth were higher than the current ones because of the absence of a significant ozone atmosphere (Cnossen et al. 2007). UV-B is higher in terrestrial environments compared

Electronic supplementary material The online version of this article (https://doi.org/10.1007/s10725-020-00639-8) contains supplementary material, which is available to authorized users.

Raúl Cassia

raul.cassia@gmail.com

1 Instituto de Investigaciones Biológicas, Facultad de Ciencias Exactas y Naturales, Universidad Nacional de Mar del PlataConsejo Nacional de Investigaciones Científicas y Técnicas, CC1245 (7600) Mar del Plata, Argentina to the water column (Rozema et al. 2002). High UV-B doses damages DNA, proteins, lipids, cell membranes, photosynthetic machinery and induces the production of reactive oxygen species (ROS). This affects plant cell integrity and viability, leading to growth retardation and to a decrease in crop yield and quality (Jordan 1996; Brosché and Strid 2003; Frohnmeyer and Staiger 2003). Consequently, plants evolved mechanisms to avoid UV-B damage during the colonization of exposed habitats (Tilbrook et al. 2013). Ancient photosynthetic organisms like cyanobacteria and various eukaryotic algae, use mycosporine-like amino acids (MAAs) as protective compounds against UV-B (Llewellyn and Airs 2010; Rastogi and Incharoensakdi 2013; Rozema et al. 2002). Land plants co-evolved with environmental UV-B levels, and the complexity of UV-B absorbing molecules increased accordingly from algae to higher plants (Rozema et al. 2002). Low levels of UV-B absorbing compounds in phyAphyB mutants of Arabidopsis thaliana are 
linked to inhibition of the Photosystem II activity after UV-B irradiation (Vladimir et al. 2020). This suggests a role of phytochromes in UV-B protection, giving more complexity to UV-B response in higher plants. Recently, Thomas and Puthur (2020) described that UV-B priming in rice seeds enhances seedlings tolerance to this radiation by the increase in UV absorbing compounds and cuticular wax content.

The protein UV RESISTANCE LOCUS (UVR8) was identified as the specific UV-B receptor in plants (Brown et al. 2005). UVR8 signaling significantly contributes to UV-B acclimation responses and the establishment of UV-B tolerance. UVR8 regulates metabolic and developmental processes and induces physiological and photomorphogenic responses. The usually UVR8-mediated UV-B responses are the inhibition of hypocotyl growth and the accumulation of flavonoids and anthocyanins. However, nowadays it is suggested that additional physiological responses are modulated by UVR8: phototropism, thermomorphogenesis, circadian clock, auxin signaling, defense, salt stress tolerance, shade avoidance, chloroplast development, stomatal opening, leaf development and downward leaf curling (for a review see Yin and Ulm 2017).

In the absence of UV-B, the UVR8 homodimer is mainly located in the cytoplasm. In the nucleus, the E3 Ubiquitin ligase COP1 (CONSTITUTIVELY PHOTOMORPHOGENIC 1) represses the activity of the transcription factor HY5 (ELONGATED HYPOCOTYL 5) (Favory et al. 2009). Following UV-B irradiation, UVR8 rapidly monomerizes and interacts in the nucleus with COP1 and WRKY36. This complex avoids the degradation of HY5, and triggers the UV-B regulated gene expression, leading to plant acclimation and stress tolerance (Heijde and Ulm 2012). One of the HY5-regulated genes is CHS (chalcone synthase), the enzyme that catalyzes the first step of flavonoid biosynthesis (Jenkins 2014; Yonekura-Sakakibara et al. 2019). Other HY5-upregulated genes are the Repressor of UV-B photomorphogenesis 1 and 2 (RUP1 and RUP2). Interaction of UVR8 with these proteins facilitates UVR8 dimerization, and subsequent inactivation (Heijde and Ulm 2012; Ulm and Jenkins 2015). UVR8 also interacts with BES1 (BRI1-EMSSUPPRESSOR1) and BIM1 (BES1-INTERACTING MYCLIKE 1), inhibiting the brassinosteroid responsive genes, and reducing the hypocotyl elongation (Sun and Zhu 2018).

UVR8 is the first photoreceptor described that perceives UV-B through tryptophan residues instead of a prosthetic chromophore (O'Hara and Jenkins 2012; Ulm and Jenkins 2015).Two Arabidopsis UVR8 (AtUVR8) high-resolution crystal structures have been determined using different crystallization conditions. However, they proved to be nearly identical in tertiary and quaternary structure (Christie et al. 2012; Wu et al. 2012; Yang et al. 2016; Zeng et al. 2015). AtUVR8 contains a core domain that forms a sevenbladed $\beta$-propeller and a flexible $\mathrm{C}$-terminal region of approximately 60 amino acids that contains a $\mathrm{C} 27$ region. Both the $\beta$-propeller domain and the $\mathrm{C}$-terminal $\mathrm{C} 27$ domain of UVR8 are necessary and sufficient for interacting with COP1. Moreover, UVR8 interacts also with WRKY36 by its C-terminal (aminoacids 397 to 440) (Yang et al. 2018). AtUVR8 has 14 tryptophan residues, 7 of which are exposed to the dimer interface. It also contains three conserved pentapeptide repeats with the motif "GWRHT" in blades 5, 6 , and 7. This motif generates a closely clustered triad of tryptophans (W233, W285 and W337) which are the most important for UV-B photoreception (Christie et al. 2012; Wu et al. 2012; Zeng et al. 2015). Structural and mutagenesis studies show a primary role for W285 and W233 in UV-B perception, whereas W337 is not essential in this process (Christie et al. 2012; Sun and Zhu 2018; Wu et al. 2012). The motif "GWRHT" from blade 6 contains W285 and is conserved in all UVR8 homologs analyzed (Fernandez et al. 2016; Han et al. 2019).

Zea mays also known as corn, is a cereal grain of agronomic importance, and has been used as a model organism in basic and applied research for nearly a century (Strable and Scanlon 2009). Previous work shows that increased UV-B radiation produces a significant reduction in dry matter accumulation and, consequently, affects yield. Moreover, an increase in flavonoid accumulation, a decrease in chlorophyll content in leaves and a reduction in protein level, sugar and starch of maize seeds have also been reported (Gao et al. 2004). This effect is reverted by salicylic acid seed priming, which diminishes the accumulation of ROS and upregulates antioxidants defenses in maize seedlings, reducing UV-B toxicity and improving growth (Singh et al. 2015). In 2011, Casati et al. described a transiently upregulation and subsequent downregulation of two UVR8-like genes upon UV-B exposure in maize leaves (Casati et al. 2011a, b). However, these genes have little homology to AtUVR8 as they were identified by homology to rice genome.

Although UVR8 is conserved, and sequences for this gene are found in all the Viridiplantae (Fernandez et al. 2016; Han et al. 2019), a few UVR8 homologs have been cloned and reported to be functional from green algae, moss and dicotyledonous. Up to now, there is no evidence of UVR8 photoreceptor from monocotyledonous, with confirmed functionality. Here, we report the molecular cloning, sequence and functional complementation of $Z m U V R 8$, the UV-B receptor of $Z$. mays.

\section{Material and methods}

\section{Plants material and growth conditions}

Seeds of A. thaliana Landsberg erecta (Ler) were used as wild-type (WT). Arabidopsis Ler and $u v r 8-1$ null mutant 
seeds (Cloix et al. 2012) were kindly provided by Dr. Gareth Jenkins (University of Glasgow, Scotland). Seeds were surface sterilized in 30\% (v/v) commercial bleach for one minute, rinsed with distilled sterile water and stratified for $72 \mathrm{~h}$ at $4{ }^{\circ} \mathrm{C}$ in darkness. Germinated seedlings were grown on agar plates containing half-strength Murashige and Skoog (MS) salts or in soil/perlite/vermiculite $(3: 1: 1, \mathrm{v} / \mathrm{v})$ under white light $\left(160 \mu \mathrm{mol} \mathrm{m} \mathrm{m}^{-2} \mathrm{~s}^{-1}\right.$, fluorescent tubes) and longday regime (light/dark: $16 / 8 \mathrm{~h}$ ) at $25^{\circ} \mathrm{C}$ in an environment controlled chamber.

Maize (Z. mays B73 inbreed line) seeds were kindly provided by Dr. Sofía Eugenia Olmos (INTA Pergamino, Argentina). Seeds were surface sterilized with $30 \%$ (v/v) commercial bleach for $20 \mathrm{~min}$ and rinsed in distilled water. Subsequently, seeds were germinated on water saturated filter paper in Petri dishes for 4-5 days and maintained at 25 ${ }^{\circ} \mathrm{C}$. Germinated seedlings were grown on soil/ vermiculite $(3: 1, \mathrm{v} / \mathrm{v})$ under a long-day regime (light/dark: $16 / 8 \mathrm{~h})$ at 25 ${ }^{\circ} \mathrm{C}$ in an environment controlled chamber with white light at $160 \mu \mathrm{mol} \mathrm{m}{ }^{-2} \mathrm{~s}^{-1}$. The topmost leaf from V6 developmental stage (when sixth leaf are visible in the leaf whorl) plants was used for experiments.

\section{Generation of transgenic Arabidopsis plants expressing ZmUVR8}

The full length coding sequence from $Z m U V R 8$ was amplified by PCR using the maize full-length EST ZM_ $\mathrm{BFb} 0066 \mathrm{P} 22 . r$ (Arizona Genomics Institute) as template, and the specific primers ZmUVR8-Fw and ZmUVR8Rv (Supplementary Table 1). The amplified cDNA was cloned into the entry pENTR/D-TOPO vector and confirmed sequence, orientation and reading frame by DNA sequencing (Macrogen). The obtained entry clone was recombined with the Gateway pH7FWG2 binary destination vector for $35 \mathrm{~S}$-driven expression in plants, with C-terminal fusion to eGFP (35S::ZmUVR8-eGFP) (Karimi et al. 2002). This vector was introduced in the Agrobacterium strain GV3101 by electroporation (Koncz and Schell 1986). Transformation into $u v r 8-1$ mutant Arabidopsis was performed by floral dip (Clough and Bent 1998). Transformants were selected based on its ability to survive on half-strength MS medium supplemented with $1 \%$ sucrose containing $15 \mathrm{mg} \mathrm{L}^{-1}$ hygromycin. Resistant seedlings were then transferred to soil and grown under conditions described above.

The transgenic lines generated were shown to have the transgene integrated at a single genetic locus through segregation analysis. From six transgenic lines T1, we obtained two homozygous independent lines (T2,\#6.5 and T3,\#5.1.7) by self-crossing. These lines were used for the experiments. The level of transgene expression in each line was examined by qRT-PCR and immunoblot.

\section{UV-B treatments}

For experiments involving UV-B light treatments, Arabidopsis seedlings were exposed $2 \mathrm{~h}$ to white light $(100 \mu \mathrm{mol}$ $\mathrm{m}^{-2} \mathrm{~s}^{-1}$, fluorescent tubes) supplemented with $3.47 \mu \mathrm{mol}$ $\mathrm{m}^{-2} \mathrm{~s}^{-1}$ narrowband UV-B (Philips TL 100W/01) in a controlled environment chamber. This dose is similar to the radiation measured from sunlight at noon in Mar del Plata summer $\left(38.0055^{\circ} \mathrm{S}, 57.5426^{\circ} \mathrm{W}\right)$.

Maize plants were exposed for 0,2 and $4 \mathrm{~h}$ to white light (100 $\mu \mathrm{mol} \mathrm{m}^{-2} \mathrm{~s}^{-1}$, fluorescent tubes) supplemented with $8.81 \mu \mathrm{mol} \mathrm{m}{ }^{-2} \mathrm{~s}^{-1}$ narrowband UV-B (Philips TL 100W/01) in a controlled environment chamber. Similar doses were used by other authors (Rius et al. 2016; Fina et al. 2017).

The spectral irradiance was determined with an UV-B photo-radiometer (Delta ohm HD2102.1).

\section{Expression analysis}

For gene expression quantification, plant materials were harvested, frozen in liquid nitrogen and grounded under RNase-free conditions. Total RNA was extracted using TRIzol method, and treated with DNase I (Invitrogen) at $37{ }^{\circ} \mathrm{C}$ for $30 \mathrm{~min}$, following the manufacturer's instructions. Then, the RNA was reverse-transcribed using the M-MLV reverse transcriptase (Thermo) following the manufacturer's instructions. cDNA obtained was used for quantitative RT-PCR using Power SYBR Green PCR mix and a StepOne machine (Applied Biosystems). Primers used are listed in Supplementary Table 1 . PCR conditions were: $10 \mathrm{~min}$ at $95^{\circ} \mathrm{C}, 40$ cycles of $15 \mathrm{~s}$ at $95^{\circ} \mathrm{C}$ and $1 \mathrm{~min}$ at $60{ }^{\circ} \mathrm{C}$. After amplification, a melting curve analysis was performed, which resulted in a single product specific melting curve. Negative controls for cDNA synthesis and qRT-PCR reactions were included in all cases. LineReg program was employed for the analysis of gene expression (Ruijter et al. 2009). The transcript relative quantifications were determined from the ratio between the starting concentration value of analyzed mRNA and the reference genes actin2 for Arabidopsis samples or thioredoxin-like (Thr) for maize as previously reported (Casati and Walbot 2004). The data shown are representative of at least three independent experiments.

For protein assays, leaves were harvested into liquid nitrogen and proteins extracted in $100 \mathrm{mM}$ buffer $\mathrm{KPO}_{4}$ (pH 7.4), 1 mM EDTA and a cocktail of protease inhibitors for plant extracts. This cocktail contains inhibitors of serine proteases, cysteine proteases, aspartyl proteases, metalloproteases and aminopeptidases (Sigma P9599). The homogenate was centrifuged for $10 \mathrm{~min}$ a $10,000 \times \mathrm{g}$ at $4{ }^{\circ} \mathrm{C}$. Protein concentration was determined by a Bradford assay. $30 \mu \mathrm{g}$ of total protein were loaded for ZmUVR8 protein expression and separated by $12 \%$ denaturing SDS-PAGE. Immunoblots were incubated with monoclonal anti-GFP (BAbCO) 
A
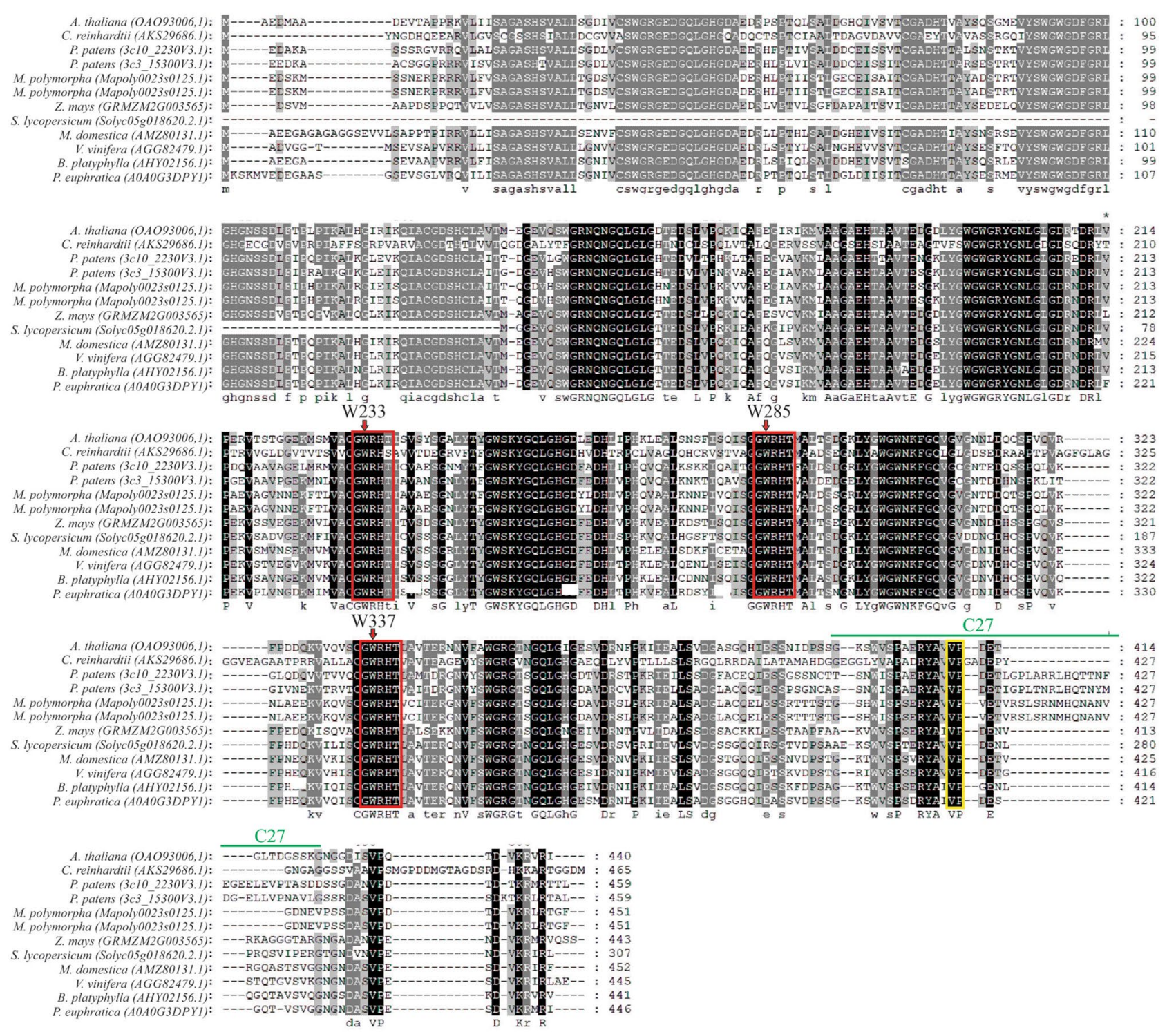

B

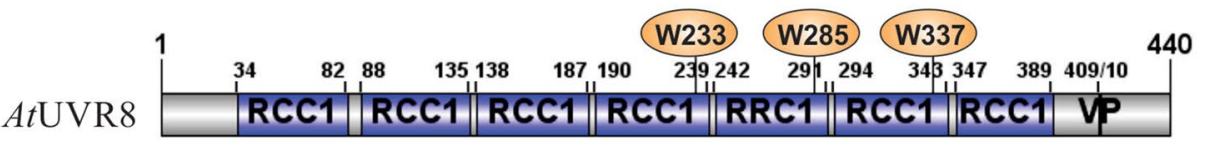

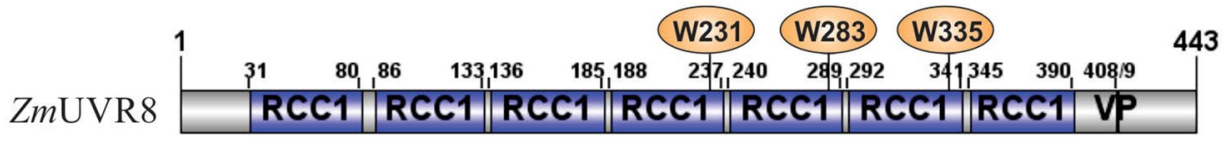

as primary antibody, gently provided by Dr. Gareth Jenkins (University of Glasgow, Scotland). After several washes, a secondary anti-mouse antibody conjugated to alkaline phosphatase and developed by NBT/BCIP staining. The membranes were stained with Ponceau S to reveal the Rubisco large subunit (rbcL) as loading control.

\section{ZmUVR8-eGFP subcellular localization}

Fifteen day old plants irradiated $1 \mathrm{~h}$ with white light or white light supplemented with UV-B $\left(3.47 \mu \mathrm{mol} \mathrm{m}^{-2} \mathrm{~s}^{-1}\right)$, were vacuum infiltrated with $5 \mu \mathrm{g} \mathrm{mL}^{-1}$ of Hoechst 33342 nucleic acid stain (Invitrogen Molecular Probes) in buffer 
4Fig. 1 ZmUVR8 sequence and structural domains. a Multiple sequence alignment of AtUVR8 homologs. Maize UVR8 (ZmUVR8) protein sequence and sequences of proteins that restore the loss-offunction of the $u v r 8-1$ null mutant were aligned with MAFFT (http:// mafft.cbrc.jp/alignment/server/) and edited with GeneDoc (Nicholas and Nicholas 1997). Red boxes indicate "GWRHT" motifs and the VP domain. The accession number of each sequence is given next to the species name. Conserved residues common to all sequences are shadowed in black and less identity is shown in gray scale. Capital letter indicates $100 \%$ of homology whereas lowercase indicates minor identity. The yellow box shows the "VP" domain in the C27 domain, important for interaction with COP1 and RUP proteins. b Schematic representation of structural domains of AtUVR8 and ZmUVR8 proteins. Analysis of amino-acid sequences was performed at the National Center for Biotechnology Information (NCBI) database. RCC1 (pfam00415), Regulator of chromosome condensation (RCC1) repeat. Arrows indicate significant tryptophans in UV-B perception (W233, 285 and 337). VP Valine-Proline domain. (Color figure online)

PBS, $0.2 \%$ Triton-X 100 for 4 min and maintained in shake at $50 \mathrm{rpm}$ and darkness for $1 \mathrm{~h}$. Then, samples were washed three times with PBS. The subcellular localization of eGFP and Hoechst 33342 was visualized by a confocal laser scanning microscope (Nikon-C1siR Eclipse TiU) under oil (Biopack) with a $\times 40$ objective. Images were taken using the Nikon EZ-C1 3.90 software. eGFP and Hoechst were excited using an argon laser at $488 \mathrm{~nm}$ and a laser at $408 \mathrm{~nm}$, respectively. eGFP emission was collected between 515 and $530 \mathrm{~nm}$ to avoid crosstalk with chloroplast autofluorescence. Hoechst 33342 fluorescence was collected at $440 / 50 \mathrm{~nm}$. The same microscope settings for GFP and Hoechst 33342 detection were used before and after UV-B illumination. Colocalization analysis was performed on two independent transgenic lines. The data shown are representative of at least three independent experiments.

\section{Hypocotyl length measurement}

Seedlings were grown for 5 days on agar plates of half strength MS salts containing 1\% sucrose in white light or white light supplemented with $3.47 \mu \mathrm{mol} \mathrm{m} \mathrm{m}^{-2} \mathrm{~s}^{-1}$ UV-B. Photographs were taken after treatments and hypocotyl lengths were measured using the ImageJ software (http:// rsb.info.nih.gov/ij). Three independent biological replicates were performed for all experiments using al least 10 seedlings for each replicate $(\mathrm{n} \geq 30)$.

\section{Bioinformatic analysis}

Multiple sequence alignments were performed using MAFFT server (http://mafft.cbrc.jp/alignment/server/) and edited with GeneDoc (Nicholas and Nicholas 1997).

\section{Results}

\section{An AtUVR8 homolog with a conserved structure is found in maize}

UVR8 homolog has not been reported in monocotyledonous. To this end, the Arabidopsis protein sequence (AAD43920.1) was used as query in a PSI-BLASTp for searching the UVR8 homolog in maize, using the NCBI (www.ncbi.nlm.nih.gov/) and Phytozome databases (www. phytozome.jgi.doe.gov/pz/portal.html). One sequence was found (GRMZM2G003565), herein named "ZmUVR8". Figure 1a shows the comparison between ZmUVR8 and the other functional UVR8 homologs. ZmUVR8 has 443 amino acids length, a calculated molecular mass of 47.15 $\mathrm{kDa}$, and $73 \%$ of identity to AtUVR8. Key tryptophan residues responsible of UV-B perception (W233, 285 and 337) are conserved in ZmUVR8, as well as the VP domain in the $\mathrm{C} 27$ region, involved in the interaction with COP1 and RUP (Fig. 1a). Figure $1 \mathrm{~b}$ shows that $Z m U \mathrm{UVR} 8$ has the same domain profile as AtUVR8, including the conserved tryptophans, the $\mathrm{C} 27$ domain and the seven repeated $\mathrm{RCC} 1$ domains.

Blastp analysis in Table 1 shows that sequences with high percentage of identity with the components of the Arabidopsis UVR8 signaling pathway were found in maize: COP1 (70\%), HY5 (68\%), HYH (48\%), RUP1 (45\%), RUP2 (46\%), WRKY36 (35\%), BES1 (50\%) and BIM1 (41\%). These results suggest a degree of conservation between the UVR8 signaling cascade of Arabidopsis, a dicotyledonous, and maize, a monocotyledonous species.

\section{UVR8 and CHS expression are regulated by UV-B in Arabidopsis and maize}

UVR8 expression was analyzed by RT-PCR in Arabidopsis and maize plants irradiated with 3.47 and $8.81 \mu \mathrm{mol} \mathrm{m} \mathrm{m}^{-2}$ $\mathrm{s}^{-1}$ of UV-B respectively. Figure 2 shows that after $2 \mathrm{~h}$ of UV-B irradiation, UVR8 expression was reduced 3 -fold in Arabidopsis and 6-fold in maize, respectively. Figure 2 also shows that $C H S$ expression was increased in both species after UV-B. These results indicate a similar regulation of $U V R 8$ and $C H S$ expression under UV-B treatment both in Arabidopsis and maize plants.

\section{Transgenic ZmUVR8 expression in the uvr8-1 null mutant}

For insights into the in vivo role of $Z m U V R 8$, we cloned the $Z m U V R 8$ cDNA into the pH7FWG2 plant expression vector driven by the CaMV-35S promoter and fused to eGFP 
Table 1 Identification of maize homologs to the Arabidopsis UVR8 signaling pathway

\begin{tabular}{llllll}
\hline Arabidopsis protein & Query ID & Zea mays identification & Identity (\%) & E-value & Accession \\
\hline COP1 & NP_180854.1 & Ubiquitin ligase protein COP1 & 70 & 0.0 & AQK75277.1 \\
HY5 & NP_001330553.1 & Uncharacterized protein LOC100286123 & 68 & $9.00 \mathrm{E}-48$ & NP_001152483.1 \\
HYH & NP_850605.1 & Uncharacterized protein LOC100286123 isoform X1 & 48 & $3.00 \mathrm{E}-27$ & XP_008643871.1 \\
RUP1 & NP_200038.1 & WD repeat-containing protein RUP2 & 45 & $5.00 \mathrm{E}-89$ & PWZ22039.1 \\
RUP2 & NP_568435.2 & WD repeat-containing protein RUP2 & 46 & $2.00 \mathrm{E}-91$ & AQK67844.1 \\
WRKY36 & NP_564976.1 & Probable WRKY transcription factor 31 & 35 & $4.00 \mathrm{E}-47$ & XP_008655458.1 \\
BES1 & NP_973863.1 & BES1/BZR1 protein & 50 & $7.00 \mathrm{E}-69$ & NP_001151195.2 \\
BIM1 & NP_001190259.1 & Unknown & 41 & $3.00 \mathrm{E}-49$ & ACN34591.1 \\
\hline
\end{tabular}

Proteins involved in UVR8 signaling cascade from Arabidopsis were used as bait in BLASTp analysis restricting the search to Z. mays and the non-redundant protein sequences database from NCBI

(35S::ZmUVR8-eGFP). This construct was used to transform the Arabidopsis uvr8-1 null mutant. After repeated selection on hygromycin and microscopy eGFP-screening, two independent T2 and T3 homozygous lines (\#6.5 and \#5.1.7 respectively) were obtained. ZmUVR8 expression was determined by quantitative RT-PCR using specific primers (Supplementary Fig. S1) that did not amplify AtUVR8 (results not shown). Figure 3 a illustrates that both lines expressed ZmUVR8 mRNA under standard growth conditions without UV-B. Line \#6.5 reached the highest level. Immunoblot with anti-GFP antibody in Fig. 3b shows that ZmUVR8-eGFP was present in both transgenic lines (the raw image is provided as the Supplementary Fig. S3). Supplementary Figure $\mathrm{S} 2$ shows no positive GFP signal in WT lines, ensuring the specificity of $Z m U V R 8$-eGFP detection. Figure 3c shows that $Z m U$ VR8 has no detrimental effects on plant development, because no evident phenotypic differences were found among WT, $u v r 8-1$, \#6.5 and \#5.1.7, after 30 days growing in standard conditions.

The sub-cellular localization of ZmUVR8-eGFP, was analyzed using a confocal laser scanning microscope. Hoechst 33342 staining was employed for nuclear staining. Figure 4 shows that ZmUVR8-eGFP fusion was mainly localized in nuclei of \#6.5 and \#5.1.7 lines, irrespective of UV-B treatments.

\section{ZmUVR8 complements the Arabidopsis uvr8-1 null mutant}

The hypocotyl length inhibition was used to analyze ZmUVR8 functionality. Figure $5 \mathrm{a}, \mathrm{b}$ shows no differences among hypocotyl length of Arabidopsis WT, uvr8-1 and transgenic line \#6.5 grown under white light. Figure $5 \mathrm{~b}$ shows that, under UV-B irradiation, the reduction in hypocotyl length was 54\% in WT plants. In contrast, the reduction in $u v r 8-1$ mutant was only $31 \%$. However, hypocotyl elongation was reduced $50 \%$ in line \#6.5, similar to WT plants. Although line \#5.1.7. showed reduction in hypocotyl length when white light was supplemented with UV-B, the response in white light was similar to that obtained after UV-B treatment in both WT and line \#6.5.

These results indicate that ZmUVR8 restores the $u v r 8-1$ null mutant phenotype under UV-B radiation, showing that it is a positive regulator in UV-B induced photomorphogenesis.

\section{ZmUVR8 promotes $\mathrm{CHS}$ and $H Y 5$ gene expression in uvr8-1 Arabidopsis mutant}

As the behaviour of line \#6.5 in the hypocotyl elongation assay is more similar to WT than line \#5.1.7, we continued working with transgenic line \#6.5.

To see if ZmUVR8 is able to induce $H Y 5$ and $C H S$, 3-weeks-old WT, $u v r 8$ - 1 and \#6.5 transgenic line plants were irradiated with white light or white light supplemented with UV-B for 2 h. HY5 and CHS expression was determined by quantitative RT-PCR. Figure 5 shows that, under white light irradiation, HY5 (C) and CHS (D) have low basal expression in all plants. After UV-B irradiation, the expression of both genes was increased in WT plants, but the $u v r 8-1$ mutant was impaired in this response. UV-B-irradiated \#6.5 line increased HY5 and CHS expression (Fig. 5c, d). These results, demonstrate that $Z m U V R 8$ was able to trigger the synthesis of UVR8-regulated UV-B absorbing compunds.

\section{Discussion}

The UV-B photoreceptor UVR8 regulates several plant responses to UV-B (Yin and Ulm 2017). UVR8 sequences from the green algae Chlamydomonas reinhardtii (Tilbrook et al. 2016), the moss Physcomitrella patens (Soriano et al. 2018), the liverworth Marchantia polymorpha (Soriano et al. 2018) and the dicotyledonous Solanum lycopersicum ( $\mathrm{Li}$ et al. 2018a), Musca domestica (Zhao et al. 2016), Vitis vinifera (Liu et al. 2014), Betula platyphylla (Li et al. 2018b) and Populus euphratica (Mao 


\section{Arabidopsis}

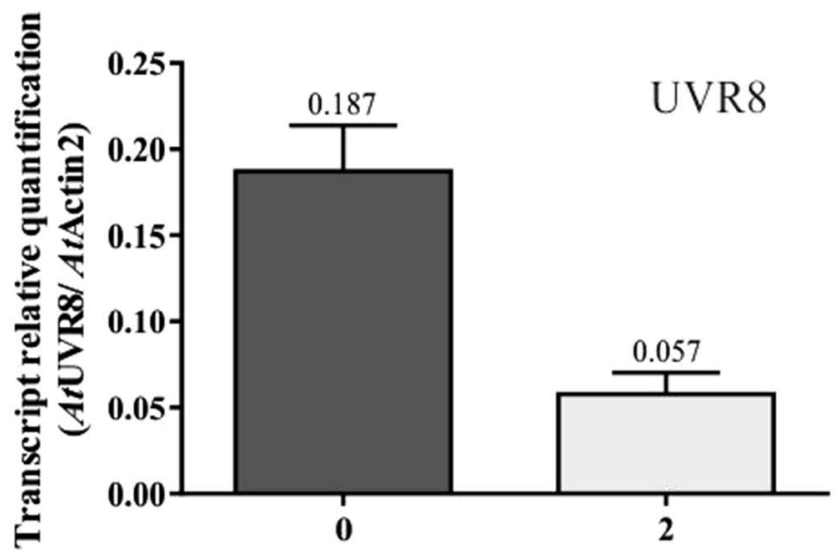

UV-B irradiation time (h)

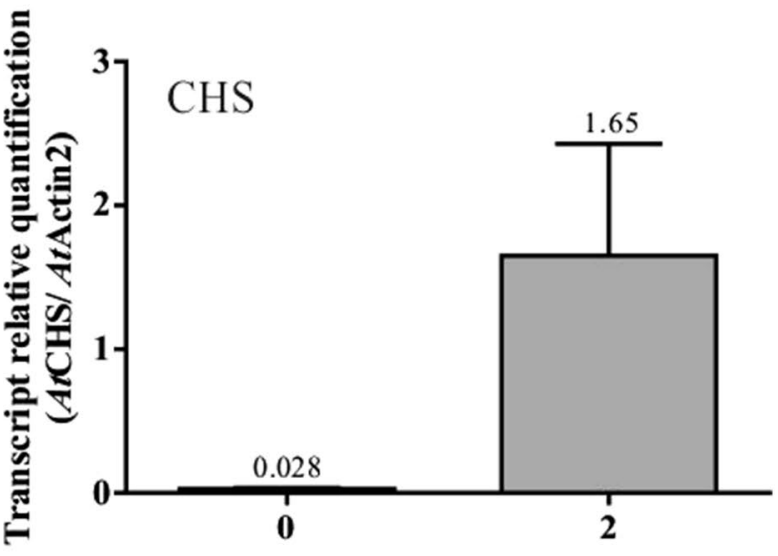

UV-B irradiation time (h)

\section{Maize}

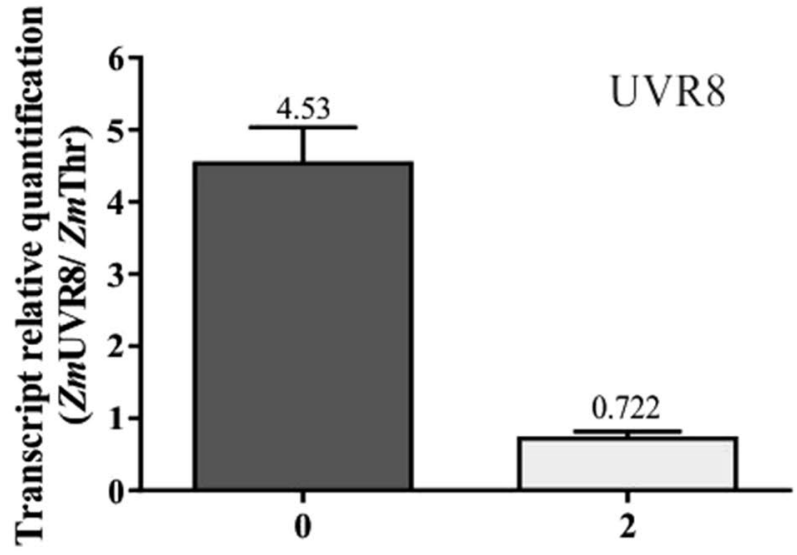

UV-B irradiation time (h)

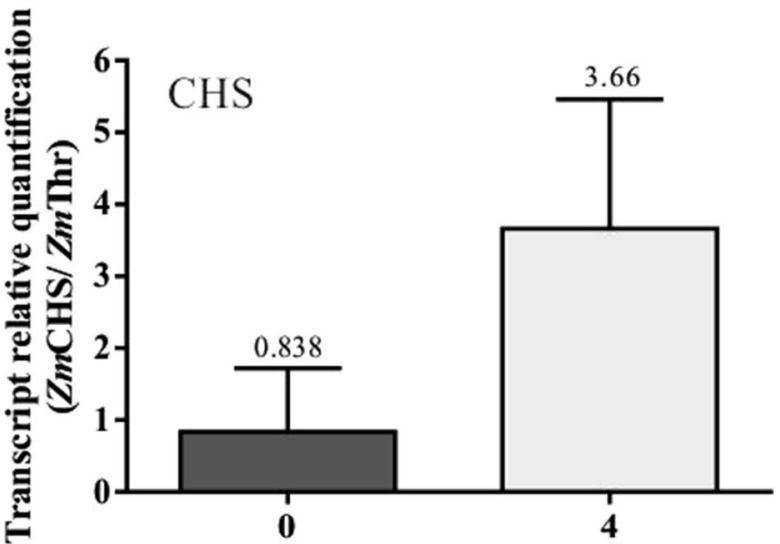

UV-B irradiation time (h)
Fig. 2 Expression of $\boldsymbol{U V R \boldsymbol { R }}$ and $\boldsymbol{C H S}$ in response to UV-B in Arabidopsis and maize plants. UVR8 and $C H S$ transcript levels were analyzed by qRT-PCR. Three week-old Arabidopsis and maize plants were irradiated during 2 and $4 \mathrm{~h}$ with 3.47 and $8.81 \mu \mathrm{mol} \mathrm{m} \mathrm{m}^{-2} \mathrm{~s}^{-1}$ of UV-B respectively. Non-irradiated plants were used as control.

et al. 2015) have been cloned, characterized, and shown to restore the loss-of-function of the UVR8 null mutant $u v r 8$ 1 (Kliebenstein et al. 2002). Two maize UV-B-responsive genes were previously reported as UVR8-like homologs by comparison with rice genome (Casati et al. 2011a, b). However, these genes have less than $34 \%$ of identity with AtUVR8, and tryptophans 233 and 285, involved in UV-B perception, are not conserved. After the maize sequence was completed, we found ZmUVR8 and, by phylogenetic studies, we concluded that a unique UVR8 sequence was present in maize (Fernandez et al. 2016). Moreover, phylogenetic studies demonstrate that UVR8, SPAs and HY5
Expression of Actin2 and thioredoxin-like (Thr) were used for Arabidopsis and maize normalization respectively. Primers are listed in Supplementary Table 1. Error bars indicate the standard deviation over three biological replicates $(n=3)$

proteins are conserved from chlorophytes to angiosperms (Fernandez et al. 2016; Han et al. 2019).

Although $U V R 8$ was reported as constitutive in Arabidopsis, UVR8 transcript decreased both in Arabidopsis and maize leaves after UV-B radiation. Moreover, comparison of ZmUVR8 expression with other functional homologues (Supplementary Table 2), indicates the existence of different regulatory mechanisms for $U V R 8$ expression in different plant species and tissues. Little is known about different regulatory mechanisms for UVR8 expression. Wu et al. (2016) proposed that UV-B enhances the production of $\mathrm{H}_{2} \mathrm{O}_{2}$, thus increasing the level of $\mathrm{NO}$ to further magnify the UVR8 


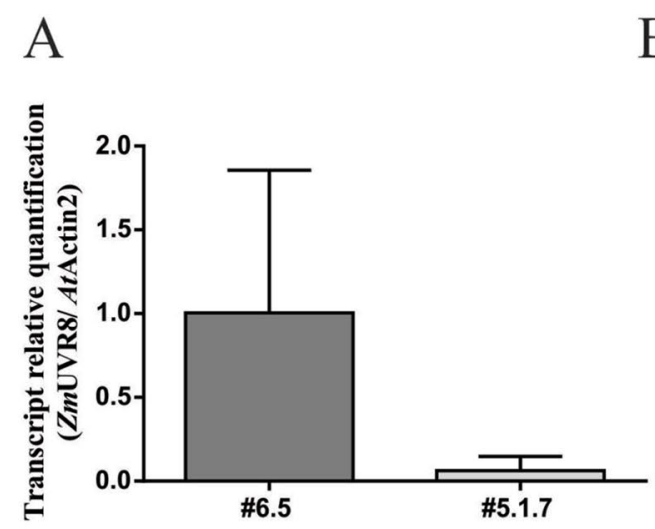

B

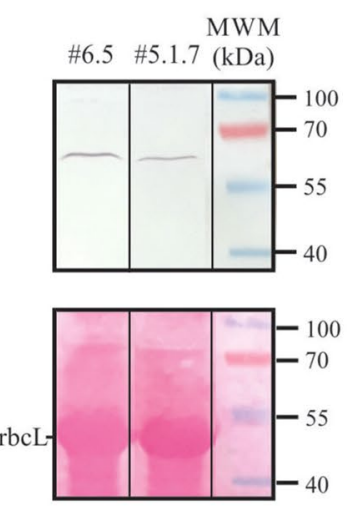

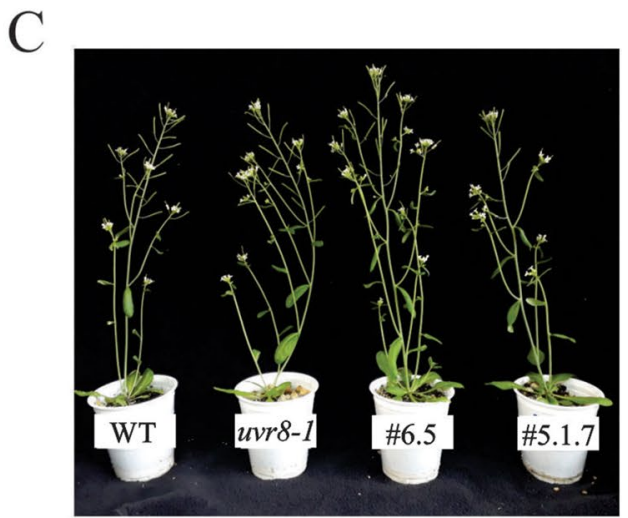

Fig. 3 35S::ZmUVR8-eGFP expression analysis in two Arabidopsis homozygous lines. a Leaves from 3-weeks-old transgenic Arabidopsis plants were used to analyze $Z m U V R 8$ transgene transcript level by qRT-PCR. Expression of Actin2 was used for normalization. Primers are listed in Supplementary Table 1. Error bars indicate the standard deviation over three biological replicates. b Denaturating immunob- lot from Arabidopsis transgenic lines \#6.5 and \#5.1.7 protein extracts (upper panel). Immunodetection was performed using anti-GFP antiserum. Stained Rubisco large subunit (rbcL) is shown as a loading control (lower panel). c Thirty-days-old WT, uvr8-1 mutant and \#6.5 and \#5.1.7 transgenic Arabidopsis lines

to nucleus, as well as for UVR8 signaling in response to UV-B. Moreover, it was demonstrated that the presence of UVR8 in the nucleus is necessary but not sufficient for its function. Kaiserli and Jenkins (2007) fused a NLS to GFP-UVR8 and observed that the constitutive nuclear localization of NLS-GFP-UVR8 is insufficient to promote HY5 expression in the absence of UV-B. Therefore, even though $Z m U V R 8$ has nuclear localization irrespective of UV-B treatment, this radiation is necessary for downstream signaling as previously reported for $A t \mathrm{UVR} 8$.

UV-B irradiation is not a mere stress signal but can also serve as an environmental stimulus to direct growth and development. A well-established UV-B morphogenic effect is the reduction of hypocotyl elongation (Kim et al. 1998). The hypocotyl growth of the uvr8-1 mutant seedlings, in stark contrast to wild-type seedlings, was not inhibited by UV-B (Favory et al. 2009). Expression of WT AtUVR8 fused to GFP or yellow fluorescent protein (YFP) in $u v r 8-1$ plants, restores the WT phenotype (Favory et al. 2009; Huang et al. 2014; O'Hara and Jenkins 2012). This response was established as a parameter of complementation by functional UVR8 homologs (Kondou et al. 2019; Li et al. 2018a, b; Liu et al. 2014; Mao et al. 2015; Soriano et al. 2018; Tilbrook et al. 2016; Zhao et al. 2016).

ZmUVR8 restored the impaired UV-B hypocotyl growth suppression, and the CHS and HY5 expression in $u v r 8-1$, working as an effective component of the UVR8 pathway. These results demonstrate that similar signaling responses to UV-B are present in monocotyledonous and dicotyledonous plants. Consequently, a canonical UVR8 pathway could be present in monocotyledonous. 


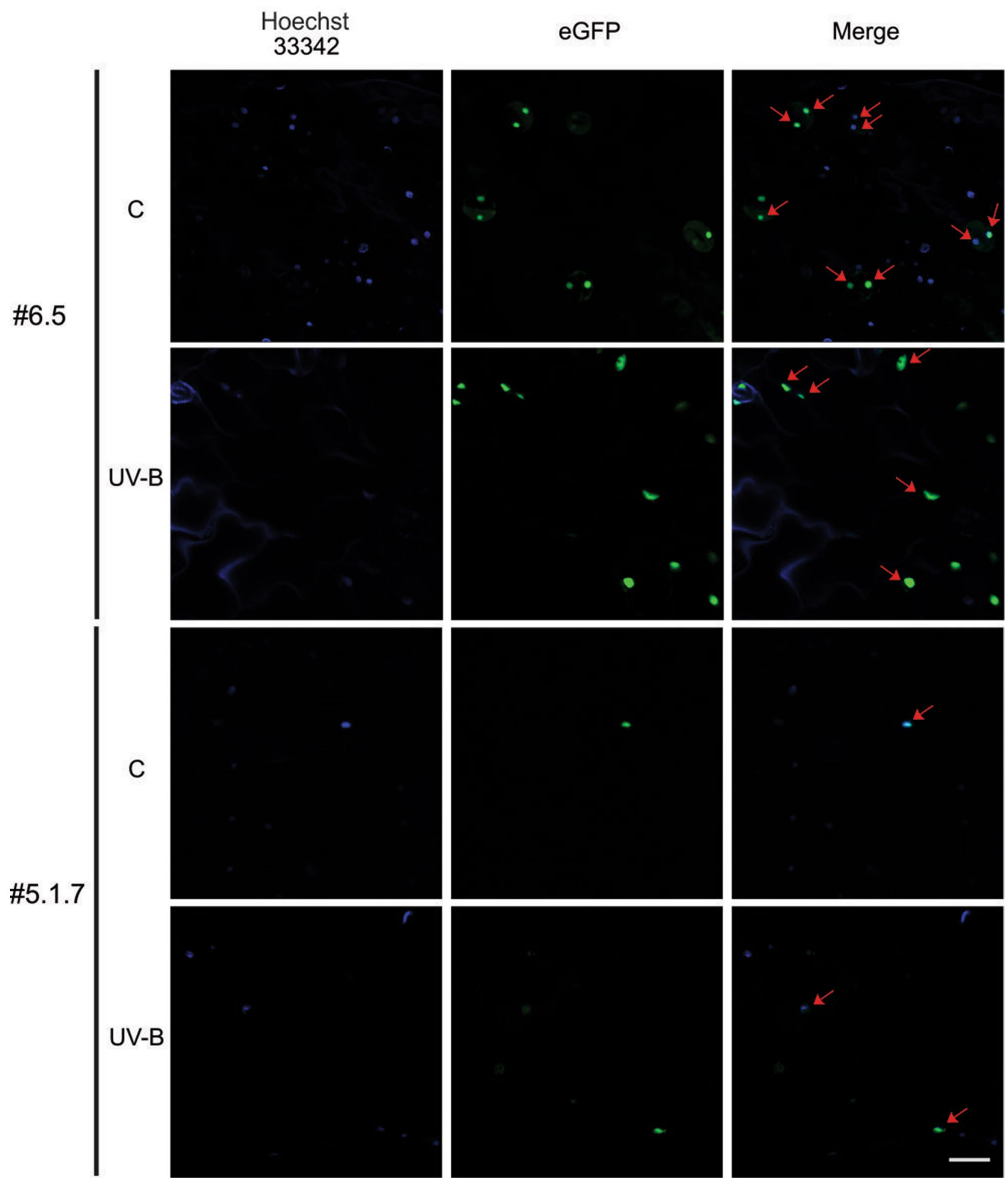

Fig. 4 Subcellular localization of eGFP-ZmUVR8. Cellular localization of ZmUVR8-eGFP fusions revealed by confocal laser microscopy. Two-weeks-old Arabidopsis plants were irradiated with 100 $\mu \mathrm{mol} \mathrm{m} \mathrm{s}^{-2}$ of white light (C), or white light plus $3.47 \mu \mathrm{mol} \mathrm{m} \mathrm{m}^{-2} \mathrm{~s}^{-1}$ UV-B (UV-B) for $1 \mathrm{~h}$. Hoechst 33342 was vacuum infiltrated to specifically stain nuclei. eGFP and Hoechst were excited using a laser at $488 \mathrm{~nm}$ and $408 \mathrm{~nm}$, respectively. eGFP emission was collected between 515 and $530 \mathrm{~nm}$ to avoid crosstalk with chloroplast autofluorescence. Hoechst 33342 fluorescence was collected at 440/50 $\mathrm{nm}$. The same microscope settings for GFP and Hoechst 33342 detection were used before and after UV-B illumination. Bar $25 \mu \mathrm{m}$. \#6.5 and \#5.1.7: transgenic Arabidopsis lines expressing ZmUVR8-eGFP. Arrows indicate co-localization 
A

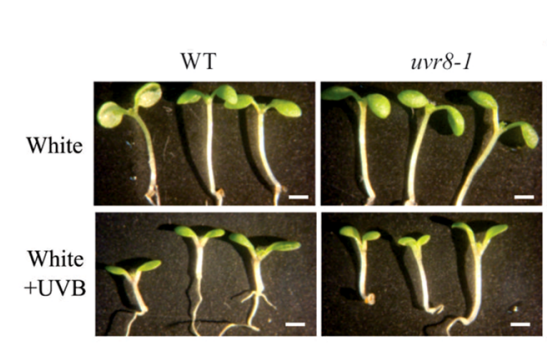

C

HY5

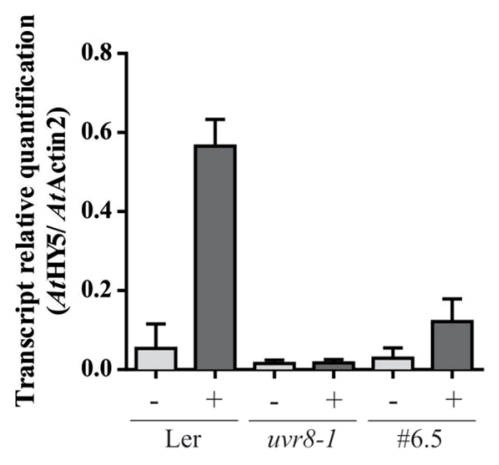

Fig. 5 Functional complementation assay of eGFP-ZmUVR8 expressed in Arabidopsis uvr8-1 mutant. a Phenotypes of the Arabidopsis WT, uvr8-1, and \#6.5 and \#5.1.7 seedling lines grown under $100 \mu \mathrm{mol} \mathrm{m} \mathrm{m}^{-2}$ of white light, or white light plus $3.47 \mu \mathrm{mol} \mathrm{m}$ $\mathrm{s}^{-1}$ of UV-B. The scale bar represents $1 \mathrm{~mm}$. Photographs were taken after treatments. b The hypocotyl lengths were measured using the ImageJ software (http://rsb.info.nih.gov/ij). Grey bars: white light, black bars: white light plus UV-B. Error bars indicate standard deviation. Different letters show significant differences between treatments

\section{Conclusion}

Since its discovery in Arabidopsis by Brown et al. (2005), cumulative evidence allows us to consider the UVR8 as a established pathway in the plant UV-B response. Although the UVR8 gene is found in all the Viridiplantae, only a handful of functional UVR8 proteins have been cloned and characterized. Moreover, a UVR8 homolog in monocotyledonous has not been reported until now. This work presents the characteristics and functionality of ZmUVR8, the UVR8 gene from maize. ZmUVR8 showed a conserved structure, and its expression was downregulated by UV-B. uvr8-1 mutants complemented with ZmUVR8 regulated the hypocotyl elongation and the expression of CHS and HY5, two of the main UVR8 responsive genes. In conclusion, this study demonstrates that $Z$. mays express a functional UVR8 protein. Our results reinforce the importance of this photoreceptor as an UV-B-responsive regulator in land plants. Further work is necessary to describe
B

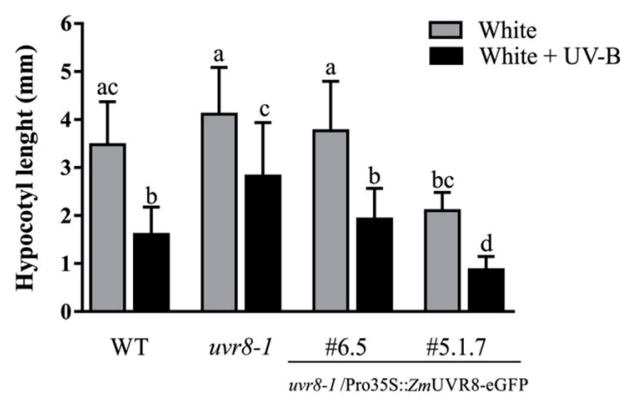

CHS

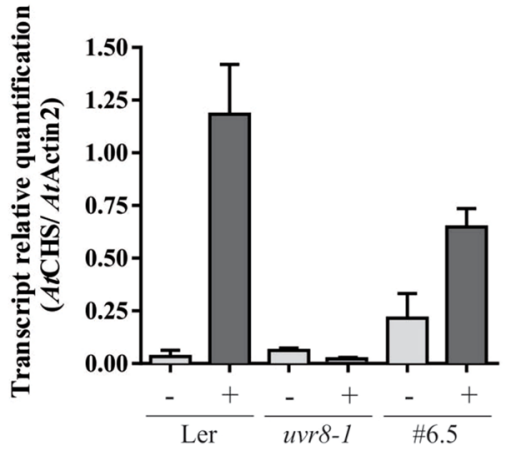

(Kruskal-Wallis one-way ANOVA on ranks. Multiple comparisons: Dunn's method, $\mathrm{p}<0.05)$. Three independent biological replicates were performed, $\mathrm{n} \geq 30$. c and $\mathbf{d}$ Three-weeks-old WT, $u v r 8-1$ and \#6.5 transgenic line plants were exposed to white light (-), or white light supplemented with $3.47 \mu \mathrm{mol} \mathrm{m} \mathrm{m}^{-2} \mathrm{~s}^{-1}$ of UV-B for $2 \mathrm{~h}(+)$. The expression of HY5 (c) and CHS (d) in leaves was determined by qRT-PCR. Expression of Actin2 was used for normalization. Error bars indicate the standard deviation of at least two biological replicates

more functional UVR8 homologs, and to discover how this pathway is regulated in response to UV-B and other abiotic stresses.

Acknowledgements We acknowledge Dr. Rius from CEFOBI Institute for technical support with maize samples.

Author contributions MF conducted experiments, interpreted data, drew figures, and collaborated in writing the manuscript. RC conceived the project and wrote the paper. LL supervised and improved the manuscript.

Funding This study was funded by the Agencia Nacional de Promoción de Ciencia y Técnica (ANPCYT) Grant Number 3589, and Universidad Nacional de Mar del Plata Grant Number EXA 818/17. LL and RC are Permanent Researcher of CONICET Argentina. MBF has Post-doctoral Fellowship of Comisión de Investigaciones Científicas (CIC).

\section{Compliance with ethical standards}

Conflict of interest The authors declare that they have no conflict of interest. 


\section{References}

Brosché M, Strid A (2003) Molecular events following perception of ultraviolet-B radiation by plants. Physiol Plant 117:1-10

Brown BA, Cloix C, Jiang GH, Kaiserli E, Herzyk P, Kliebenstein DJ, Jenkins GI (2005) A UV-B-specific signaling component orchestrates plant UV protection. Proc Natl Acad Sci USA 102:18225-18230. https://doi.org/10.1073/pnas.0507187102

Casati P, Walbot V (2004) Rapid transcriptome responses of maize (Zea mays) to UV-B in irradiated and shielded tissues. Genome Biol 5:R16. https://doi.org/10.1186/gb-2004-5-3-r16

Casati P, Campi M, Morrow DJ, Fernandes JF, Walbot V (2011) Transcriptomic, proteomic and metabolomic analysis of UV-B signaling in maize. BMC Genomics 12:321. https://doi. org/10.1186/1471-2164-12-321

Casati P, Morrow DJ, Fernandes JF, Walbot V (2011) Rapid maize leaf and immature ear responses to UV-B radiation. Front Plant Sci 2:33. https://doi.org/10.3389/fpls.2011.00033

Christie JM et al (2012) Plant UVR8 photoreceptor senses UV-B by tryptophan-mediated disruption of cross-dimer salt bridges. Science 335:1492-1496. https://doi.org/10.1126/science.1218091

Cloix C et al (2012) C-terminal region of the UV-B photoreceptor UVR8 initiates signaling through interaction with the COP1 protein. Proc Natl Acad Sci USA 109:16366-16370. https://doi. org/10.1073/pnas.1210898109

Clough SJ, Bent AF (1998) Floral dip: a simplified method for Agrobacterium-mediated transformation of Arabidopsis thaliana. Plant J 16:735-743

Cnossen I, Sanz-Forcada J, Favata F, Witasse O, Zegers T, Arnold NF (2007) Habitat of early life: solar X-ray and UV radiation at earth's surface $4-3.5$ billion years ago. Geophys Res Lett 112:E2

Favory JJ et al (2009) Interaction of COP1 and UVR8 regulates UV-Binduced photomorphogenesis and stress acclimation in Arabidopsis. EMBO J 28:591-601. https://doi.org/10.1038/emboj.2009.4

Fernandez MB, Tossi V, Lamattina L, Cassia R (2016) A comprehensive phylogeny reveals functional conservation of the UV-B photoreceptor UVR8 from green algae to higher plants. Front Plant Sci 7:1698.https://doi.org/10.3389/fpls.2016.01698

Fina J, Casadevall R, AbdElgaward H, Prinsen E, Markakis MN, Beemster GTS, Casati P (2017) UV-B inhibits leaf growth through changes in growth regulating factors and gibberellin levels. Plant Physiol 174:1110-1126. https://doi.org/10.1104/pp.17.00365

Frohnmeyer H, Staiger D (2003) Ultraviolet-B radiation-mediated responses in plants. Balancing damage and protection. Plant Physiol 133:1420-1428. https://doi.org/10.1104/pp.103.030049

Gao W, Zheng Y, Slusser JR, Heisler GM, Grant RH, Xu J, He D (2004) Effects of supplementary ultraviolet-B irradiance on maize yield and qualities: a field experiment. Photochem Photobiol 80:127-131. https://doi.org/10.1562/2004-05-03-RA-156.1

Han X, Chang X, Zhang Z, Chen H, He H, Zhong B, Deng XW (2019) Origin and evolution of core components responsible for monitoring light environment changes during plant terrestrialization. Mol Plant 12:847-862. https://doi.org/10.1016/j.molp.2019.04.006

Heijde M, Ulm R (2012) UV-B photoreceptor-mediated signalling in plants. Trends Plant Sci 17:230-237. https://doi.org/10.1016/j. tplants.2012.01.007

Heilmann M, Jenkins GI (2013) Rapid reversion from monomer to dimer regenerates the ultraviolet-B photoreceptor UV RESISTANCE LOCUS8 in intact Arabidopsis plants. Plant Physiol 161:547-555. https://doi.org/10.1104/pp.112.206805

Huang X, Yang P, Ouyang X, Chen L, Deng XW (2014) Photoactivated UVR8-COP1 module determines photomorphogenic UV-B signaling output in Arabidopsis. PLoS Genet 10:e1004218. https://doi.org/10.1371/journal.pgen.1004218
Jenkins GI (2014) The UV-B photoreceptor UVR8: from structure to physiology. Plant Cell 26:21-37. https://doi.org/10.1105/ tpc.113.119446

Jordan BR (1996) The effects of ultraviolet-B radiation on plants: a molecular perspective. Adv Bot 22:98-138

Kaiserli E, Jenkins GI (2007) UV-B promotes rapid nuclear translocation of the Arabidopsis UV-B specific signaling component UVR8 and activates its function in the nucleus. Plant Cell 19:2662-2673. https://doi.org/10.1105/tpc.107.053330

Karimi M, Inze D, Depicker A (2002) GATEWAY vectors for Agrobacterium-mediated plant transformation. Trends Plant Sci 7:193-195

Khudyakova AY et al (2019) Impact of UV-B radiation on the photosystem II activity, pro-/antioxidant balance and expression of light-activated genes in Arabidopsis thaliana hy 4 mutants grown under light of different spectral composition. J Photochem Photobiol B 194:14-20. https://doi.org/10.1016/j.jphotobiol .2019 .02 .003

Kim BC, Tennessen DJ, Last RL (1998) UV-B-induced photomorphogenesis in Arabidopsis thaliana. Plant J 15:667-674

Kliebenstein DJ, Lim JE, Landry LG, Last RL (2002) Arabidopsis UVR8 regulates ultraviolet-B signal transduction and tolerance and contains sequence similarity to human regulator of chromatin condensation 1. Plant Physiol 130:234-243. https://doi. org/10.1104/pp.005041

Koncz C, Schell J (1986) The promoter of TL-DNA gene 5 controls the tissue-specific expression of chimaeric gene carried by a novel type of Agrobacterium binary vector. Mol Gen Genet 204:383-396

Kondou Y et al (2019) Physiological function of photoreceptor UVR8 in UV-B tolerance in the liverwort Marchantia polymorpha. Planta 249:1349-1364. https://doi.org/10.1007/s00425-019-03090 $-\mathrm{w}$

Li H et al (2018) Tomato UV-B receptor SIUVR8 mediates plant acclimation to UV-B radiation and enhances fruit chloroplast development via regulating SIGLK2. Sci Rep 8:6097. https://doi. org/10.1038/s41598-018-24309-y

$\mathrm{Li}$ X et al (2018) Molecular cloning and functional analysis of a UV-B photoreceptor gene, BpUVR8 (UV Resistance Locus 8), from birch and its role in ABA response. Plant Sci 274:294-308. https ://doi.org/10.1016/j.plantsci.2018.06.006

Liu L, Gregan S, Winefield C, Jordan B (2014) From UVR8 to flavonol synthase: UV-B-induced gene expression in Sauvignon blanc grape berry. Plant Cell Environ 38:905-919. https://doi. org/10.1111/pce.12349

Llewellyn CA, Airs RL (2010) Distribution and abundance of MAAs in 33 species of microalgae across 13 classes. Mar Drugs 8:12731291. https://doi.org/10.3390/md8041273

Mao K, Wang L, Li YY, Wu R (2015) Molecular cloning and functional analysis of UV RESISTANCE LOCUS 8 (PeUVR8) from Populus euphratica. PLoS ONE 10:e0132390. https://doi.org/10.1371/ journal.pone. 0132390

Nicholas KB, Nicholas HBJ (1997) GeneDoc: a tool for editing and annotating multiple sequence alignments

O'Hara A, Jenkins GI (2012) In vivo function of tryptophans in the Arabidopsis UV-B photoreceptor UVR8. Plant Cell 24:37553766. https://doi.org/10.1105/tpc.112.101451

Pearson WR (2014) An introduction to sequence similarity ("homology") searching. Curr Protoc Bioinform. https://doi. org/10.1002/0471250953.bi0301s42

Rajhi I et al (2011) Identification of genes expressed in maize root cortical cells during lysigenous aerenchyma formation using laser microdissection and microarray analyses. N Phytol 190:351-368. https://doi.org/10.1111/j.1469-8137.2010.03535.x

Rastogi RP, Incharoensakdi A (2013) UV radiation-induced accumulation of photoprotective compounds in the green alga 
Tetraspora sp. CU2551. Plant Physiol Biochem 70:7-13. https:// doi.org/10.1016/j.plaphy.2013.04.021

Razeghi J, Kianianmomeni A (2019) UV-B response is modulated by cell-type specific signaling pathway in multicellular green algae Volvox carteri. Plant Growth Regul 87:303-315. https://doi. org/10.1007/s10725-018-0472-7

Ren $\mathrm{H}$ et al (2019) Two E3 ligases antagonistically regulate the UV-B response in Arabidopsis. Proc Natl Acad Sci USA. https://doi. org/10.1073/pnas.1816268116

Rius SP, Emiliani J, Casati P (2016) P1 epigenetic regulation in leaves of high altitude maize landraces: effect of UV-B radiation. Front Plant Sci 7:523. https://doi.org/10.3389/fpls.2016.00523

Rizzini L et al (2011) Perception of UV-B by the Arabidopsis UVR8 protein. Science 332:103-106. https://doi.org/10.1126/scien ce. 1200660

Rozema J et al (2002) The role of UV-B radiation in aquatic and terrestrial ecosystems - an experimental and functional analysis of the evolution of UV-absorbing compounds. J Photochem Photobiol B 66:2-12

Ruijter JM, Ramakers C, Hoogaars WM, Karlen Y, Bakker O, van den Hoff MJ, Moorman AF (2009) Amplification efficiency: linking baseline and bias in the analysis of quantitative PCR data. Nucleic Acids Res 37:e45. https://doi.org/10.1093/nar/gkp045

Singh VP et al (2015) Role of salicylic acid-seed priming in the regulation of chromium(VI) and UV-B toxicity in maize seedlings. Plant Growth Regul 78:79-91. https://doi.org/10.1007/s1072 5-015-0076-4

Soriano G, Cloix C, Heilmann M, Nunez-Olivera E, Martinez-Abaigar J, Jenkins GI (2018) Evolutionary conservation of structure and function of the UVR8 photoreceptor from the liverwort Marchantia polymorpha and the moss Physcomitrella patens. N Phytol 217:151-162. https://doi.org/10.1111/nph.14767

Strable J, Scanlon MJ (2009) Maize (Zea mays): a model organism for basic and applied research in plant biology. Cold Spring Harb Protoc. https://doi.org/10.1101/pdb.emo132

Sun K, Zhu Z (2018) Illuminating the nucleus: UVR8 interacts with more. Trends Plant Sci 23:279-281. https://doi.org/10.1016/j. tplants.2018.03.002

Thomas TTD, Puthur JT (2020) UV-B priming enhances specific secondary metabolites in Oryza sativa (L.) empowering to encounter diverse abiotic stresses. Plant Growth Regul. https://doi. org/10.1007/s10725-020-00628-x

Tilbrook K et al (2016) UV-B perception and acclimation in Chlamydomonas reinhardtii. Plant Cell. https://doi.org/10.1105/ tpc. 15.00287

Tilbrook K, Arongaus AB, Binkert M, Heijde M, Yin R, Ulm R (2013) The UVR8 UV-B photoreceptor: perception, signaling and response. Arabidopsis Book 11:e0164. https://doi.org/10.1199/ tab.0164

Ulm R, Jenkins GI (2015) Q\&A: how do plants sense and respond to UV-B radiation? BMC Biol 13:45. https://doi.org/10.1186/s1291 5-015-0156-y

Vladimir D et al (2020) Linking sensitivity of photosystem II to UV-B with chloroplast ultrastructure and UV-B absorbing pigments contents in A. thaliana L. phyAphyB double mutants. Plant Growth Regul 91:13-21. https://doi.org/10.1007/s10725-020-00584-6

Wu D et al (2012) Structural basis of ultraviolet-B perception by UVR8. Nature 484:214-219

Wu Q, Su N, Zhang X, Liu Y, Cui J, Liang Y (2016) Hydrogen peroxide, nitric oxide and UV RESISTANCE LOCUS8 interact to mediate UV-B-induced anthocyanin biosynthesis in radish sprouts. Sci Rep 6:29164. https://doi.org/10.1038/srep29164

Yang Y et al (2018) UVR8 interacts with WRKY36 to regulate HY5 transcription and hypocotyl elongation in Arabidopsis. Nat Plants 4:98-107. https://doi.org/10.1038/s41477-017-0099-0

Yang X, Montano S, Ren Z (2016) How does photoreceptor UVR8 perceive a UV-B signal? Photochem Photobiol 91:993-1003

Yin R (2017) Cooling down thermomorphogenesis by UV-B signaling. Trends Plant Sci 22:447-449. https://doi.org/10.1016/j.tplan ts.2017.04.003

Yin R, Ulm R (2017) How plants cope with UV-B: from perception to response. Curr Opin Plant Bio 17:42-48

Yin R, Skvortsova MY, Loubery S, Ulm R (2016) COP1 is required for UV-B-induced nuclear accumulation of the UVR8 photoreceptor. Proc Natl Acad Sci USA 113:E4415-E4422. https://doi. org/10.1073/pnas.1607074113

Yonekura-Sakakibara K, Higashi Y, Nakabayashi R (2019) The origin and evolution of plant flavonoid metabolism. Front Plant Sci 10:943. https://doi.org/10.3389/fpls.2019.00943

Zeng X et al (2015) Dynamic crystallography reveals early signalling events in ultraviolet photoreceptor UVR8. Nat Plants. https://doi. org/10.1038/nplants.2014.6

Zhao C, Mao K, You CX, Zhao XY, Wang SH, Li YY, Hao YJ (2016) Molecular cloning and functional analysis of a UV-B photoreceptor gene, MdUVR8 (UV Resistance Locus 8), from apple. Plant Sci 247:115-126. https://doi.org/10.1016/j.plantsci.2016.03.006

Publisher's Note Springer Nature remains neutral with regard to jurisdictional claims in published maps and institutional affiliations. 